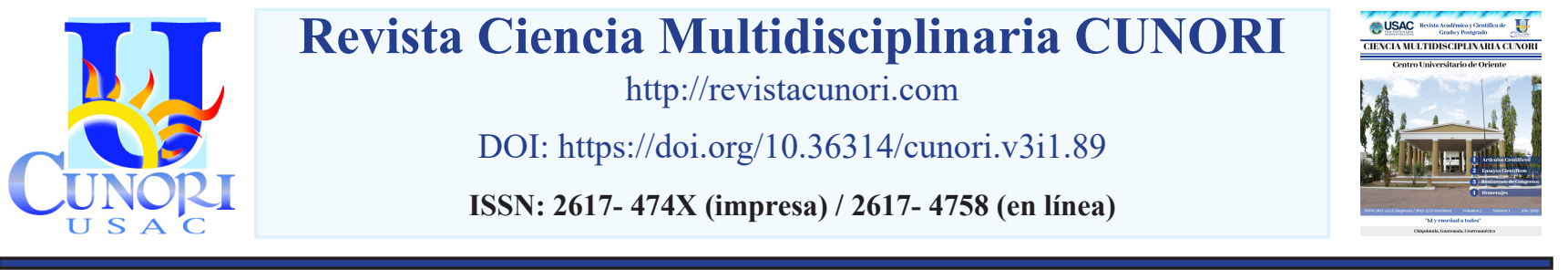

Como citar el ensayo

Lemus, F., Lemus, D., Alarcón, S., Villela, C.,(2019). Análisis de la importancia del planeamiento educativo. Revista Ciencia Multidisciplinaria CUNORI, 3(1), 135-141. DOI: https://doi.org/10.36314/cunori.v3i1.89

\title{
Análisis de la importancia del planeamiento educativo
}

\section{Analysis of the importance of educational planning}

\author{
Florinely Lemus*, Deisy Lemus, Silvia Alarcón, Claudia Villela \\ Departamento de Estudios de Posgrado, Centro Universitario de Suroriente -CUNSURORI- USAC. Jalapa \\ Recibido: 25 de noviembre de 2018 / Revisión: 13 de diciembre de 2018 / Aceptado: 01 de marzo de 2019
}

Disponible en internet el 30 de agosto de 2019

*Autor para correspondencia.

Correo electrónico: florecitalemus@hotmail.com

\section{Resumen}

Ne conoce como planificación, planeación o planteamiento, generalmente en países de habla hispana, pero en el sentido más universal, implica tener uno o varios objetivos en común, junto con acciones requeridas para concluirse exitosamente. Otras definiciones, más precisas, incluyen la planificación como un proceso de toma de decisiones para alcanzar un futuro deseado, teniendo en cuenta la situación actual y los factores internos y externos que pueden influir en el logro de los objetivos, va de lo más simple a lo complejo, dependiendo del medio a aplicarse. La acción de planear en la gestión se refiere a planes y proyectos en sus diferentes ámbitos, niveles y actitudes. Se entiende por planeamiento y sus sinónimos, a toda actividad previamente programada a su realización o aplicación, siendo un proceso de suma importancia en todo proceso de la vida, para obtener resultados positivos y alcanzar el éxito en el futuro. El objetivo del ensayo, consiste en analizar la importancia de la planificación, tanto en el ámbito universal como educativo.

Palabras clave: planeamiento, educación

\begin{abstract}
T is known as planning, planning or planning, generally in Spanish-speaking countries, but in the most universal sense, it implies having one or several objectives in common, together with actions required to conclude successfully. Other definitions, more precise, include planning as a decision-making process to reach a desired future, taking into account the current situation and the internal and external factors that can influence the achievement of objectives, going from the simplest to the complex, depending on the medium to be applied. The action of planning in management refers to plans and projects in their different areas, levels and attitudes. It is understood by planning and its synonyms, to any activity previously programmed to its realization or application, being a process of utmost importance in all life processes, to obtain positive results and achieve success in the future. The aim of the essay is to analyze the importance of planning, both in the universal and educational field.
\end{abstract}

Keywords: planning, education 


\section{Introducción}

La palabra planeamiento y su significado son extensos, por lo que el presente ensayo se encausará al planeamiento educativo. La planeación y planeamiento es un accionar que está vinculado a planear, o elaborar un plan. A través de la planeación una persona u organización se fija alguna meta y estipula qué pasos debería seguir para llegar hasta ella. Es frecuente que la planificación se inicie con la identificación de un problema y continúe con el análisis de las diferentes opciones disponibles.

El objetivo es analizar la importancia de la planificación, tanto en el ámbito universal como educativo para que en el futuro los resultados sean los deseados. La estrategia de investigación se muestra con los siguientes contenidos: planeamiento educativo, el proceso de la planeación educativa, planeación, fines de la planeación educativa, tipos de planeación educativa; tales como: planeación prospectiva, circunspectiva y estratégica, política y planeamiento de la educación, el planeamiento de la educación como proceso técnico, el planeamiento educativo como proceso político y planeamiento educativo como proceso administrativo.

El planeamiento educativo tiene una importancia esencial, ya que permite responder a las necesidades, intereses y las motivaciones que tienen los aprendientes o estudiantes, que se desprenden del proceso enseñanza aprendizaje, ya que es una herramienta para el docente para mejorar la calidad educativa. Se determinó que al aplicar las diferentes herramientas del planeamiento se fortalecen los ambientes académicos según las condiciones adecuadas para atender las necesidades educativas de los estudiantes.

\section{El planeamiento educativo}

\section{Planeación}

Se iniciará definiendo la palabra planeación, la cual se refiere a planear sobre una actividad a realizar para obtener los mejores resultados con el mínimo de tiempo y de recursos. Es el resultado de hacer planes; es la acción de analizar una determinada situación, sus antecedentes y expectativas, para establecer los objetivos, políticas y la relación de actividades necesarias para que un sistema cumpla con sus necesidades.

Planeación es la aplicación racional de la mente humana en la toma de decisiones anticipatoria, con base en el conocimiento previo de la realidad, para controlar las acciones presentes y prever sus consecuencias futuras, encausadas al logro de un objetivo plenamente deseado y satisfactorio. La planeación es la determinación de lo que va a hacerse, incluye decisiones de importancia, como el establecimiento de políticas, objetivos, redacción de programas, definición de métodos específicos, procedimientos y el establecimiento de las células de trabajo y otras más. Se deduce que, toda vez que el individuo quiera obtener resultados alentadores de lo que desea llevar a cabo, este con anticipación debe llevar a cabo el orden de las acciones que pretende realizar, y tener claros los objetivos que desea lograr, a esto se le denomina, planeamiento, planeación o planificación.

\section{Planeamiento Educativo}

Se presentan las principales tendencias del planeamiento y lo que de ellas ha tomado la educación específicamente, haciendo hincapié en los procesos de racionalidad, es decir, encontrando la o las lógicas 
del pensamiento, de la acción, de las prácticas del planeamiento en cada paradigma. Esto último implica el análisis epistemológico subyacente en cada postura para avanzar hacia un posicionamiento crítico y participativo. Los paradigmas en juego en el ámbito educativo a lo largo de la década de 1960 van adquiriendo cuerpo la idea de planeamiento educativo en América Latina; en sus inicios surge como un enfoque normativo o reactivo caracterizado por el reduccionismo ilegítimo de todas las acciones a comportamientos y la eliminación artificial del otro según (Andretich, 2008). Y por otra parte (Matus, 1987) hace referencia a la planificación, desde esta perspectiva tradicional, es concebida como una metodología y un conjunto de técnicas especializadas instrumentadas por expertos y destinadas a establecer los mecanismos normativos para alcanzar los fines que se establecen.

Por consiguiente, al poner en práctica el planeamiento, implica una reforma para la educación, ya que es un tanto complejo, extraer a los docentes de su rutina de preparación de sus clases, para que entren a un nuevo proceso de planeación previo a realizar toda actividad educativa.

\section{El proceso de la planeación educativa}

Toda situación de enseñanza está condicionada por la inmediatez y la imprevisibilidad, por lo que la planificación permite: por un lado, reducir el nivel de incertidumbre y, por otro, anticipar lo que sucederá en el desarrollo de la clase, otorgando rigurosidad y coherencia a la tarea pedagógica en el marco de un programa. Habitualmente, la planificación funciona como una exigencia formal. En ese sentido se la reduce a un instrumento burocrático, y se le despoja de su función primordial, que es la de guía organizadora y estructuradora de la práctica, según describe el libro (planeacion de sistemas educativos, 2011).

Por consiguiente, la planeación en el proceso educativo es fundamental y funcionable, ya que esta es una herramienta útil para el docente, puesto que evita improvisaciones y errores en el desarrollo de la clase, los cuales deben de evitarse a toda costa. Según (Wittrock, 1990) en sentido general, la planeación dirigida al sistema educativo en su conjunto se expresa en un cuerpo de políticas que intentan traducirse en orientaciones para la acción de los agentes directamente involucrados en el funcionamiento del sistema escolar. No obstante, la planeación educativa como su nombre lo indica, está ligada a todas las actividades pedagógicas, en la que se benefician todos los componentes educativos, para lograr un bien común.

\section{Fines de la planeación educativa}

Según la (UNESCO, 2015). La planeación educativa se encarga de especificar los fines, objetivos y metas de la educación. Gracias a este tipo de planeación es posible definir qué hacer y con qué recursos y estrategias trabajar. No obstante, si se quiere tener la idea clara, del camino que se va a recorrer, es necesario prever la línea de acción a seguir, en otras palabras, el fin de la planeación en el ámbito educativo es llegar al lugar al cual nos dirigió la ruta trazada. En la escuela se forman seres humanos los cuales son miembros de una sociedad, dicha labor de formar es propiamente del docente, por ende, de este depende la formación y el cómo se desenvuelve el estudiante en la sociedad, tarea ardua la del docente, por lo que es indiscutible el tema de la planeación de sus clases. 


\section{Tipos de planeación educativa}

De acuerdo con (Ruiz, 2011), por concepción de planeación se entiende el enfoque a partir del cual se infiere el carácter del proceso de planeación. La clasificación propuesta es: Retrospectiva, prospectiva, circunspectiva y estratégica.

\section{Planeación retrospectiva}

Se basa en la exploración del pasado para conocer el futuro; es decir, observa el comportamiento de las variables relevantes del pasado y con base a ellas determina las posibles tendencias futuras. Existen dos modalidades de esta planeación: determinista y probabilística. La planeación determinista se basa en un modelo donde las variables a considerar son susceptibles de control. Se trata principalmente de variables endógenas que se analizan con una visión sistemática, en la cual a cada valor de entrada corresponde un sólo valor de salida. La planeación probabilística toma en cuenta variables tanto endógenas como exógenas que no están bajo control o no son controlables. Sólo es posible determinar su probabilidad de ocurrencia a partir de su comportamiento histórico. Como resultado de esta planeación se obtienen probables escenarios futuros que facilitan la selección de las mejores alternativas de acción.

\section{Planeación prospectiva}

Se enfoca al diseño de un futuro deseado ideal, totalmente libre de las restricciones que pudieran provenir de tomar en cuenta el pasado y el presente. En una etapa posterior se establecen los futuros probables que se derivan de la situación existente y, por último, se selecciona un escenario factible a la luz del futuro ideal. La distingue el énfasis en la formulación de los objetivos o futuro deseado y la búsqueda activa de medios para hacerlo posible. El proceso de planeación se extiende desde la formulación de los ideales sociales y económicos más generales, hasta los detalles de la elaboración e implantación de decisiones. Se deduce que, toda planeación prospectiva, es la que encamina al futuro soñado, en la que ordenadas las acciones de acuerdo a los ideales que se encaminarán al futuro deseado.

\section{Planeación circunspectiva}

Excluye la visión del futuro y se circunscribe a situaciones o problemas particulares del presente. En otros términos, se habla de previsión que generalmente toma acciones para dar respuesta a necesidades urgentes del presente. La planeación circunspectiva atiende a problemas del momento, tiene limitada su libertad de elección entre diferentes alternativas. Esta concepción adopta dos modalidades: coyuntural y correctiva. La planeación coyuntural se aplica cuando se presentan momentos favorables para ejecutar una acción o realizar un cambio. La planeación correctiva se utiliza para resolver problemas actuales o para corregir aspectos desfavorables.

\section{Planeación estratégica}

Este tipo de planeación contiene elementos que pudiesen hacer que se considere de ella un método; considerando que éste se refiere tanto al enfoque de la dirección, como al proceso. Los administradores consideran a la organización una unidad total y se preguntan a sí mismo qué debe hacerse a largo plazo para lograr las metas organizacionales. 
Es un proceso que sienta las bases de una actuación integrada a largo plazo, establece un sistema continuo de toma de decisiones, identifica cursos de acción específicos, formula indicadores de seguimiento sobre los resultados, e involucra a los agentes sociales y económicos locales a lo largo de todo el proceso. Otro aspecto importante es que considera que un plan debe operacionalizarse (elemento fundamental para hacer funcionar un plan) y además hace énfasis en la efectividad del mismo (finalidad de todo plan). La planeación estratégica no puede estar separada de funciones administrativas como: la organización, dirección, motivación y control.

\section{Política y planeamiento de la educación}

Según (Lozano, 1968), el planeamiento educativo como un proceso y un método para formular y ejecutar políticas de educación cuyas características se dice que comprende tanto el proceso propiamente dicho de preparación de planes como su adopción por decisiones gubernamentales y su ejecución por parte de los organismos responsables de la administración educativa en todos sus niveles. Ahora se definirá de modo más estricto el carácter del planeamiento como instrumento al servicio del proceso más amplio de gobierno y administración educativos, distinguiendo y correlacionando sus aspectos técnicos, políticos y administrativos.

Las relaciones entre planeamiento, política y administración educativas están llamadas a recibir en el futuro una mayor y más cuidadosa atención; será éste el único modo de suprimir los equívocos y las confusiones observadas con frecuencia, y asegurar así al planeamiento una vigencia real unida a mayor eficacia.

\section{El planeamiento de la educación como proceso técnico}

En sus aspectos técnicos, este planeamiento es un proceso metódico, interdisciplinario y permanente de diagnóstico de la realidad y previsión de necesidades de una comunidad o país en materia educativa, así como de determinación de las acciones y medios alternativos necesarios para satisfacerlas; en síntesis, es una metodología de análisis, previsión, programación y evaluación del desarrollo educativo. Persiguiendo este fin utiliza, adapta y combina conceptos y técnicas de la pedagogía, economía, sociología, antropología cultural, demografía, estadística, administración y otras disciplinas auxiliares relacionadas con la cuantificación y explicación de los hechos sociales.

No obstante, en Guatemala las instituciones académicas pretenden que todos los docentes del país, en todos los niveles y áreas a impartir, estén totalmente planificadas metodológicamente para evitar improvisaciones y que el estudiante reciba el conocimiento de acuerdo a las necesidades.

\section{El planeamiento educativo como proceso político}

En su aspecto político, el planeamiento se identifica con el proceso de adopción de decisiones de gobierno a través de sus órganos legislativos y ejecutivos; o dicho, en otros términos, es el proceso por el cual las alternativas y los planes preparados por los técnicos se convierten en política, orientación y norma de las actividades del Estado. En materia educativa las decisiones pueden presentar formas y contenidos muy diversos: reducirse a la formulación de propósitos y directivas generales de la acción, establecerlos de modo detallado, tener un carácter imperativo o simplemente indicativo. 
Por consiguiente, la política y la educación juegan un papel paralelamente en cuestiones de gobierno, ya que el planeamiento conlleva a la toma de decisiones por medio de sus entes competentes.

\section{El planeamiento educativo como proceso administrativo}

En sus aspectos administrativos, el planeamiento educativo lo constituyen las series coordinadas de actividades que efectúan los diversos organismos ejecutivos de la administración de esos servicios, en función y en cumplimiento de las políticas y los planes adoptados; en ese sentido, el planeamiento educativo es un proceso de actividad administrativa programada. Será necesario insistir sobre el hecho que para poco o nada servirían políticas y planes que carezcan de la posibilidad de concretarse. En último término, con el planeamiento de la educación se persigue un fin eminentemente práctico, que las iniciativas, decisiones y actuaciones tomadas al nivel de los organismos operativos, direcciones generales de educación, supervisión, administraciones provinciales y locales, dirección de establecimientos, se inserten órdenes, ejecuten y evalúen dentro del marco y la perspectiva de un plan de educación preciso y comprensivo.

Se dice que, la planificación en los procesos administrativos, fundamenta los objetivos que se pretenden alcanzar cuando se lleve a la práctica. El planeamiento en sí, es una serie de actividades coordinadas y administradas para alcanzar las competencias deseadas.

\section{Conclusión}

La planificación educativa es un proceso que implementa acciones y actividades para lograr el desarrollo pedagógico deseado. Inmersos en el contexto de planeamiento educativo, es necesario tomar en cuenta las siguientes actividades previas, tales como: fijar los grandes objetivos, elaboración de una guía o manual de trabajo para uso o servicio que se organice, el orden y secuencia de actividades a llevar a cabo, línea de acción bien definida, entre otras.

Una planeación fundamentada en la coherencia de actividades a futuro, es el cauce para la satisfacción de las necesidades y exigencias que la misma sociedad establece, ya que se debe formar para el futuro, para ser miembros activos en la sociedad, llevar al país en el que se vive a otra dimensión, salir del subdesarrollo y ser personas preparadas para asumir todo lo que en la vida diaria se presente.

Se afirma que, una planificación no debe ser objetivo de plagio, puesto que un plan no puede funcionar en otro ambiente, tiempo y lugar. El desarrollo es diferente, el nivel social y económico de igual manera varia, por lo que se recomienda establecer la planeación que satisfaga las necesidades actuales.

\section{Referencias bibliográficas}

Andretich, G. (2008). Planeamiento educativo como herramienta de democratización: una mirada desde lo epistemológico Ciencia, Docencia y Tecnología, Argentina: Universidad Nacional de Entre Ríos Concepción del Uruguay.

Lozano, S. (1968) El planeamiento de la Educación. Santiago de Chile. Editorial Universitaria, S. A. 
Matus, C. (1987). Política, Planificación y Gobierno. Caracas, Venezuela: Altadir. https://doi.org/10.18356/ e00423c2-es

Ruiz, M. (2011) "Sistemas de Planeación para Instituciones Educativas. México: Trillas.

UNESCO (2015). Principios del planeamiento de la educación. La Organización de las Naciones Unidas para la Educación, la Ciencia y la Cultura. https://doi.org/10.15381/anales.v45i3-4.6061

Wittrock, M. (1990) La investigación de la enseñanza III Profesores y alumnos, Barcelona: Paidós Educador.

\section{Sobre la autora}

\section{Florinely Lémus Pérez}

Licenciada en Pedagogía y Administración Educativa, actualmente estudia la Maestría en Docencia Universitaria con Énfasis en Andragogía en el Centro Universitario de Suroriente CUNSURORI de la USAC. Se desempeña como Directora de un colegio privado en el municipio de Ipala, Chiquimula, es también profesora en un instituto por cooperativa en el mismo municipio.

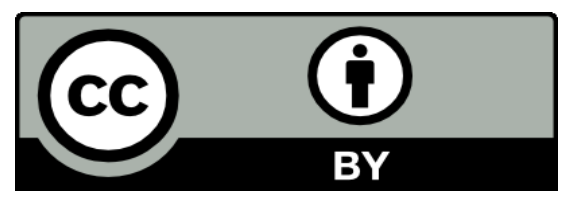

Este texto está protegido por una licencia CreativeCommons 4.0.

Usted es libre para compartir, copiar y redistribuir el material en cualquier medio o formato y adaptar el documento, remezclar, transformar y crear a partir del material para cualquier propósito, incluso comercialmente, siempre que cumpla la condición de atribución: usted debe reconocer el crédito de una obra de manera adecuada, proporcionar un enlace a la licencia, e indicar si se han realizado cambios. Puede hacerlo en cualquier forma razonable, pero no de forma tal que sugiera que tiene el apoyo del licenciante o lo recibe por el uso que hace. 